\title{
Glucose and Mannose: a Link between Hydration and Sweetness
}

\author{
N. H. Rhys ${ }^{+}$, F. Bruni ${ }^{\&}$, S. Imberti ${ }^{\dagger}$, S. E. McLain ${ }^{+}$, and M. A. Ricci\&* \\ \&Dipartimento di Scienze, \\ Sezione di Nanoscienze, \\ Università degli Studi "Roma Tre", \\ Via della Vasca Navale 84, \\ 00146 Roma, Italy. \\ $\dagger$ ISIS Neutron and Muon source, \\ STFC, Rutherford Appleton Laboratory, \\ Harwell Campus, Didcot, \\ Oxfordshire OX11 0QX, United Kingdom. \\ + Department of Biochemistry, \\ University of Oxford, \\ South Park Road, Oxford, \\ Oxfordshire OX1 3QU, UK. \\ * Corresponding author: Maria Antonietta Ricci

$$
\begin{aligned}
& \text { tel.: }+39-0657337226 \\
& \text { fax: }+39-0657337210
\end{aligned}
$$ \\ e-mail: mariaantonietta.ricci@uniroma3.it
}

(Dated:) 


\begin{abstract}
Glucose and mannose have a different degree of sweetness, implying different affinity to the sweet taste receptor. While the receptor structure is still undefined, there are several geometrical models for their binding mechanism. A detailed study of the hydration structure of sugars with known degree of sweetness is bound to provide information on the accuracy of such models. Our neutron diffraction study on the hydration of glucose and mannose show that both $\alpha$ - and $\beta$-glucose form strong hydrogen bonds with water, and that the steric hindrance of their first hydration shell matches the receptor geometrical model. The $\alpha$-anomer of mannose has a similar, well defined first hydration shell, but with fewer and weaker hydrogen bonds compared to glucose. Conversely, the hydration shell of $\beta$-mannose (reported as bitter) does not match the receptor geometrical model. These findings suggest a link between the hydration shell of sugars and their degree of sweetness.
\end{abstract}




\section{Introduction}

Among our senses, taste has the primary function of driving our appetite, protecting from poisons and contributing to the overall enjoyment of a meal. Sweet taste permits the identification of energy-rich nutrients, umami allows the recognition of amino acids, salt taste ensures the proper dietary electrolyte balance, and sour and bitter warn against the intake of potentially poisonous chemicals. In particular, the perception of sweet or bitter taste is ascribed to cells expressing the taste receptor type1 member 2 (T1R2) which forms heterodimers with the member 3 (T1R3), or the receptors type2 (T2R). Upon binding to the sweet or bitter molecule the heterodimers couple to the G-proteins to activate a signal transduction pathway that culminates, in the brain, in the release of neurotransmitters ${ }^{1}$. While a large body of information is available on the dynamical aspects of carbohydrate hydration (see for instance 2,3 and references therein), there is limited information concerning the sugar-sweet receptor dynamics of docking, given the absence of a crystallographic structure of the receptor-ligand complex. It thus remains an intriguing question as to what determines a sugar to be perceived sweeter than another, given the relatively small differences between the stereochemistry of sugars. Interestingly, the degree of sweetness of sugars does not correlate with their solubility in water, or even the viscosity of the solution ${ }^{4}$, to quote a few macroscopic properties.

The first molecular model for sweet taste was proposed by Shallenberger and Acree $^{5}$ and later improved by Kier ${ }^{6,7}$, with the definition of the sweetness triangle. According to this model, a sweet molecule needs to occupy a specific binding pocket within the receptor, where it associates with the protein via hydrogen bonds through a donor site $(\mathrm{AH})$ and an acceptor site (B); the binding-site triangle is completed by a hydrophobic (X) site. The distance between the $\mathrm{AH}$ site and the other two vertices of the triangle is thought to be around $3-4 \AA$, while the distance between $\mathrm{B}$ and $\mathrm{X}$ is about $5 \AA$. The $\mathrm{AH} / \mathrm{B} / \mathrm{X}$ structure of the sugar must correspond the $\mathrm{B} / \mathrm{AH} / \mathrm{X}$ structure on the receptor. While this model may be too simplistic to explain all the observations, its evolution, called the multipoint interaction model ${ }^{8}$, still represents a useful, unifying criterion in rationalizing structure-sweetness relationships. In particular, the Nofre and Tinti model ${ }^{8}$ elucidates the taste differences and the significant enhancement in sweetness from artificial sweeteners. According to this model, the human sweetness receptor has at least eight interaction sites available to the sugar molecules, la- 


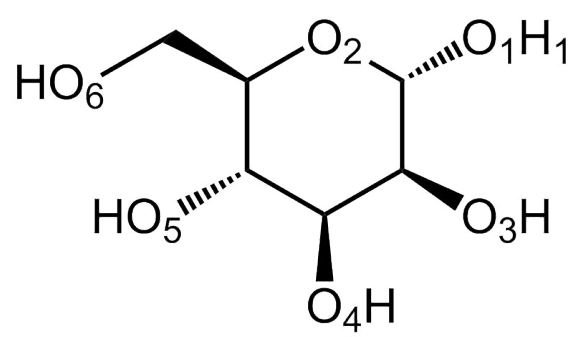

$\alpha$-mannose

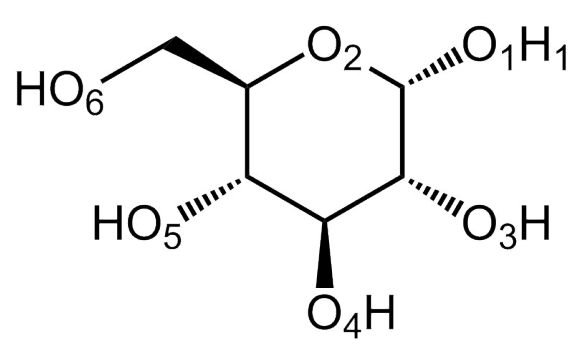

$\alpha$-glucose<smiles>OC[C@H]1O[C@H](O)[C@@H](O)[C@@H](O)[C@@H]1O</smiles>

$\beta$-mannose

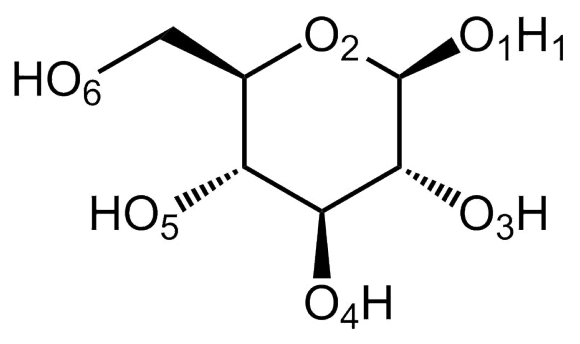

$\beta$-glucose

FIG. 1: Molecular structure of $\alpha$ and $\beta$ anomers of mannose (top row) and glucose (bottom row). The figure reports also the labels used hereafter to identify specific sites on the molecules.

belled B, AH, XH, G1, G2, G3, G4 and D, so that poor sweeteners, such as glycine, interact with only a few receptor sites, while the majority of sugars interact with more than three sites. The receptor-binding interactions take place via hydrogen bonds, at donor $(\mathrm{AH}$ and $\mathrm{XH})$ or acceptor (B, G and $\mathrm{D})$ sites, or via ionic forces (B, $\mathrm{AH}$ and $\mathrm{XH}$ sites); moreover the G sites may exploit a hydrophobic interaction.

In this context, the rationale behind our work is that an experimental determination of the hydration of sugars at the atomistic level, effectively provides a "map" of the sites preferentially available for hydrogen bonding (both donor/acceptor). This map can then be compared to the sweet receptor models, providing an experimental microscopic link between this and the functional information about sweetness perception.

Here, we present a comparison between two monosaccharides, mannose and glucose ${ }^{9}$, using a combination of neutron diffraction and simulation. In water, both sugars predominantly assume a chair conformation, with a natural proportion of $\alpha$ to $\beta$ anomers of $67: 33$ for mannose and 36:64 for glucose: the molecular structures and salient labelling for both molecules are shown in Fig. 1. It should be noted that $\mathrm{O} 2$ is often referred to as $\mathrm{O} 5$ in the 
literature given its position in the sugar molecule, here it has been labelled as $\mathrm{O} 2$ to allow for a more direct comparison between previously published work on the hydration of sugars in solution ${ }^{9,10}$ The sweetness of mannose and glucose solutions, measured with reference to sucrose, are 0.32 and 0.74 respectively (in their natural anomeric composition). Thus sensible differences in the interaction with the receptor are expected and, within our hypothesis, these should show distinct hydrogen bonding (HB) patterns. Mannose is paradigmatic in itself as $\alpha$-mannose is sweet while $\beta$-mannose can elicit a bitter aftertaste $^{11,12}$.

The atomistic hydration of small biological molecules, such as sugars, can be efficiently investigated by neutron diffraction ${ }^{10,13-17}$. This technique is particularly sensitive to the $\mathrm{O} \cdots \mathrm{H}$ correlations. The differential selectivity of neutrons for hydrogen and deuterium nuclei allows several isotopically distinct solutions to be measured, where these data are structurally equivalent, yet give rise to different diffraction patterns ${ }^{18}$. In the following the site-specific hydration of the two sugars in aqueous solutions at a concentration of 1 sugar molecule per 50 water molecules is discussed.

\section{Materials and Methods}

The experiments were performed using the SANDALS diffractometer ${ }^{19}$ (ISIS Facility, STFC, UK ${ }^{20}$ ), using standard Ti-Zr sample containers (1 mm thickness), at ambient pressure and temperature. Other aspects of glucose have been previously published in Ref. 9, where the isotopic composition of all investigated samples is reported. Mannose $\left(\mathrm{C}_{6} \mathrm{H}_{12} \mathrm{O}_{6}\right)$ was purchased from Sigma-Aldrich, labelled hereafter as H-mannose, with a portion processed through a cycle of dissolution in $\mathrm{D}_{2} \mathrm{O}$ and subsequent freeze-drying in order to obtain a powder of $\mathrm{C}_{6} \mathrm{D}_{5} \mathrm{H}_{7} \mathrm{O}_{6}$ (D5-Mannose) where each -OH hydrogen is deuterated. The two isotopically substituted powders have been dissolved in $\mathrm{H}_{2} \mathrm{O}$ and $\mathrm{D}_{2} \mathrm{O}$ in the right proportions, in order to obtain the samples listed in Table I and exploit the advantages of the isotopic substitution method ${ }^{18}$.

Each sample was measured for $\sim 8$ hours, corresponding to a total proton current at the ISIS target of $1000 \mu \mathrm{A}$. Data were also collected for empty containers, the empty instrument and a vanadium standard, in order to obtain the experimental interference differential cross sections, or $F(Q)$, shown in Fig. 2, after raw data processing through the standard Gudrun routine ${ }^{21}$. These are defined as: 
TABLE I: Labels and composition of the investigated mannose samples.

\begin{tabular}{|c|c|c|c|c|}
\hline sample label & D5-mannose (mole) & H-mannose (mole) & $\mathrm{D}_{2} \mathrm{O}$ (mole) & $\mathrm{H}_{2} \mathrm{O}$ (mole) \\
\hline $\mathrm{D}$ & 1 & 0 & 50 & 0 \\
$\mathrm{H}$ & 0 & 1 & 0 & 50 \\
$\mathrm{HD}$ & 0.5 & 0.5 & 25 & 25 \\
null & 0.36 & 0.64 & 18 & 32 \\
\hline
\end{tabular}

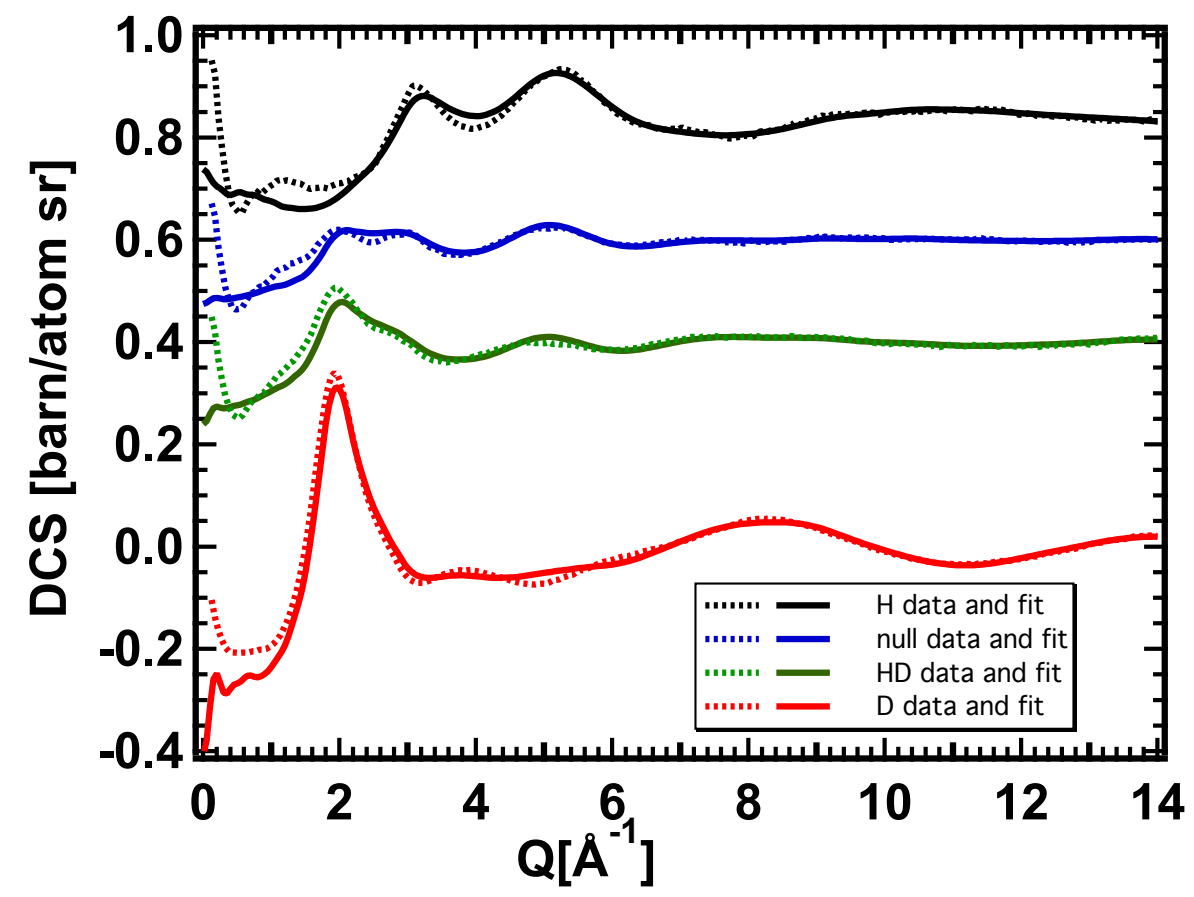

FIG. 2: (color online) The experimental $F(Q)$ for the four investigated samples of mannose solutions (dots), compared with their EPSR fit (solid lines). H, null, HD and D samples data are reported from top to bottom; data are offset for clarity.

$$
F(Q)=\sum_{i} \sum_{j \geq i} w_{i j}\left[S_{i j}(Q)-1\right]
$$

where the $w_{i j}$ coefficients weight the contribution of the individual $i-j$ pairs, accounting for their concentration and neutron scattering lengths ${ }^{22}$, and the partial structure factors, $S_{i j}(Q)$, are the Fourier transform of the site-site radial distribution functions, $g_{i j}(r)$. 
The experimental $F(Q)$ have been modelled using Empirical Potential Structure Refinement $(\mathrm{EPSR})^{23}$. This is a Monte Carlo routine, which refines the interaction potentials in a model against a set of the experimental data, from a starting configuration equilibrated using a reference potential. EPSR has proven particularly useful and reliable in extracting detailed atomistic information from neutron diffraction data collected from aqueous solutions ${ }^{9,13-16,18,24,26}$. In the present work we have equilibrated a box containing 67 $\alpha$-mannose molecules, $33 \beta$-mannose molecules and 5000 water molecules at a density of 0.1034 atoms $/ \AA^{3}$, which interact via the reference potentials reported in Table II (for water the standard Simple Point Charge/Extended, SPC/E, potential has been used ${ }^{27}$ ). The two sugar anomers have been distinguished by applying a different label to the $\mathrm{O} 1$ atomic site. After a few thousand of Monte Carlo iterations, at stable energy, we have switched on the potential refinement, in order to achieve a good fitting of the data: this is driven by the feedback from the comparison between data and simulation. Once a good fit was attained, a production run was started to accumulate the 6000 configurations used to calculate the individual $g_{i j}(r)$ and the other structural information. We remark that the $\alpha$ - and $\beta$ - anomers can only be distinguished by the EPSR simulation, while such a distinction is not possible looking only at the experimental data.

The best model obtained for mannose samples (Fig. 2), is of a similar quality as reported for glucose (Ref. 9). The residuals present at low wavevectors, $Q$, are due to an inelastic scattering background ${ }^{28}$, which is more prominent in hydrogen-containing samples. As this scattering is incoherent, it does not compromise the reliability of the structural information.

Molecular configurations recorded by EPSR have been used to evaluate the $g_{i j}(r)$ functions, which give the average interactions between $j$ and $i$ atoms at distance $r$. From the entire set of $g_{i j}(r)$ s obtained by EPSR fit to the experimental data, here the most relevant ones with respect to the candidate sites for taste-receptor binding are discussed. In addition, the spatial density functions (SDFs) obtained by spherical harmonics approximation of the EPSR configurations ${ }^{23}$ are shown along with spatial density maps (SDMs) and contact coefficients, calculated using ANGULA ${ }^{14,29-31}$. The SDFs give a 3D representation of hydration shells around the whole molecule. By highlighting the regions where the probability of finding a water molecule is higher than a chosen threshold value, the site-specific SDMs give a more quantitative measure of the occupancy of these regions via a 'heat' map which shows the relative density of neighboring molecules around a central atom in atoms $/ \AA^{-3}$. The 
TABLE II: Potential parameters used to start the EPSR simulation for the mannose solution.

\begin{tabular}{|c|c|c|c|}
\hline atom & $\epsilon(\mathrm{kJ} / \mathrm{mol})$ & $\sigma(\AA)$ & $\mathrm{q}(\mathrm{e})$ \\
\hline $\mathrm{Ow}^{a}$ & 0.6500 & 3.166 & -0.8476 \\
$\mathrm{Hw}^{b}$ & 0 & 0 & +0.4238 \\
$\mathrm{C}^{c}$ & 0.8000 & 3.700 & +0.21453 \\
$\mathrm{M}^{d}$ & 0 & 0 & +0.0537 \\
$\mathrm{O}^{e}$ & 0.6500 & 3.100 & -0.7000 \\
$\mathrm{O}_{2}{ }^{f}$ & 0.6500 & 3.100 & -0.34108 \\
$\mathrm{H}^{g}$ & 0 & 0 & +0.4356 \\
\hline
\end{tabular}

${ }^{a}$ water atomic sites are labelled $\mathrm{Ow}$ and $\mathrm{Hw}$

${ }^{b}$ water atomic sites are labelled $\mathrm{Ow}$ and $\mathrm{Hw}$

${ }^{c}$ all carbon atoms are labelled $\mathrm{C}$ and share the same initial potential parameters

${ }^{d}$ methyl hydrogens are labelled $\mathrm{M}$ and have been assigned a lower positive charge, compared to Hw.

${ }^{e}$ hydroxyl oxygens on mannose have been initially assigned the same potential parameters and are labelled here $\mathrm{O}$

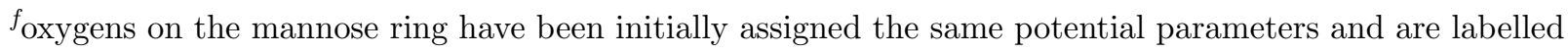
here $\mathrm{O}_{2}$

${ }^{g}$ hydroxyl hydrogens have been assigned the same parameters as water hydrogen and are labelled $\mathrm{H}$

contact coefficients give, relatively, the most probable closest contacts between any atom on the sugar molecules and the surrounding water $\mathrm{Ow}$ atoms as a probabilistic distribution. For the analysis herein, the relative contacts between the sugar hydroxyl oxygens and $\mathrm{Ow}$ have been extracted from the total probabilistic distribution of contacts between $\mathrm{O} w$ and all atoms within the different sugars, and then re-normalized solely to the other hydroxyl oxygens in order to give the relative contacts only between $\mathrm{Ow}$ and the sugar -OH groups.

\section{Results}

Inspection of Fig. 3 shows that in both sugars the $\mathrm{O} 2$ site likely plays the role of the $\mathrm{X}$ or $\mathrm{G}$ site of the receptor binding models, as the $g_{\mathrm{O} 2 \mathrm{Hw}}(r)$ s show the absence of a peak at a characteristic hydrogen bonding $(\mathrm{HB})$ distance of $\sim 1.8 \AA$, similar to what is observed for other X-O-X molecular motifs in solution ${ }^{13,17,24}$. 


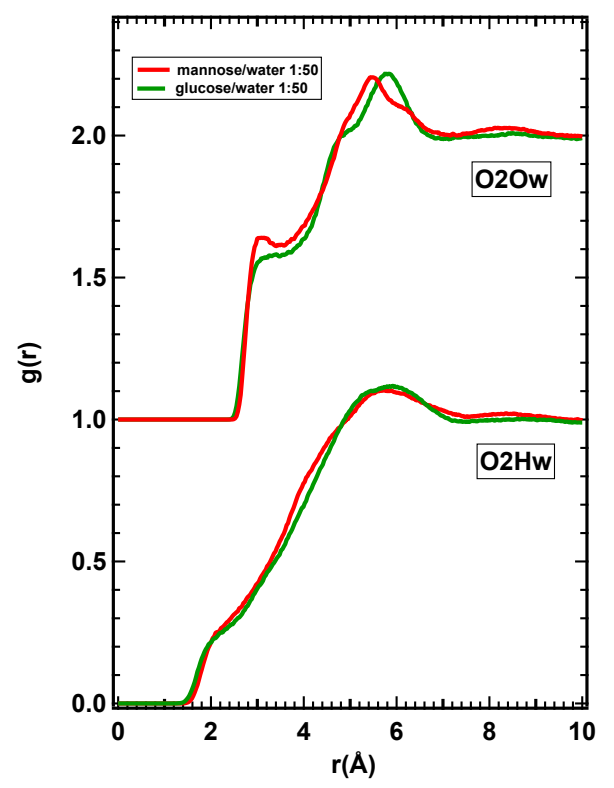

FIG. 3: (color online) The radial distribution functions for water around the oxygen atom on the ring of the two monosaccharides, O2. Data for mannose are reported in red, those for glucose in green. The $g_{O 2 O w}(r)$ functions have been offset for clarity.

In contrast, the differences between the hydration shells of mannose and glucose are clear from the hydroxyl-water $g(r) \mathrm{s}$ (see Fig. 4). In particular, in Fig. 4A, the $g_{O H w}(r)$ for mannose shows a shoulder at $1.92 \AA$ compared with the sharp peak at $1.77 \AA$ for glucose. These differences in the interaction between the hydroxyl oxygens (O3, O4, O5, O6; Fig. 1) and the water hydrogens are also observed in the O-Ow $g(r) \mathrm{s}$, where the average distance for mannose is $r_{O O w}=2.85 \AA$ compared to glucose at $2.73 \AA$ (this peak is also much broader in mannose). The $-\mathrm{OH}$ hydrogens in both monosaccharides form HBs with the surrounding water solvent, although for mannose these are somewhat weaker, as suggested by the different average nearest-neighbor distances $\left(r_{H O w}=1.89 \AA\right.$ and $1.77 \AA$, for mannose and glucose, respectively) and by the broad peak observed for mannose. This suggests that the glucose $-\mathrm{OH}$ groups interact with water as both donor and acceptor sites, contrary to the -OH groups in mannose, which only act as donors to the surrounding water solvent. Moreover, the average number of $\mathrm{HBs}$ are $\sim 0.6$ and $\sim 0.53$ for mannose, for $\mathrm{H}$ and $\mathrm{O}$, respectively, compared with $\sim 0.8$ and $\sim 0.9$ for glucose. We notice that this result contrasts with previous molecular dynamics simulations ${ }^{25}$, which was not constrained by experimental 

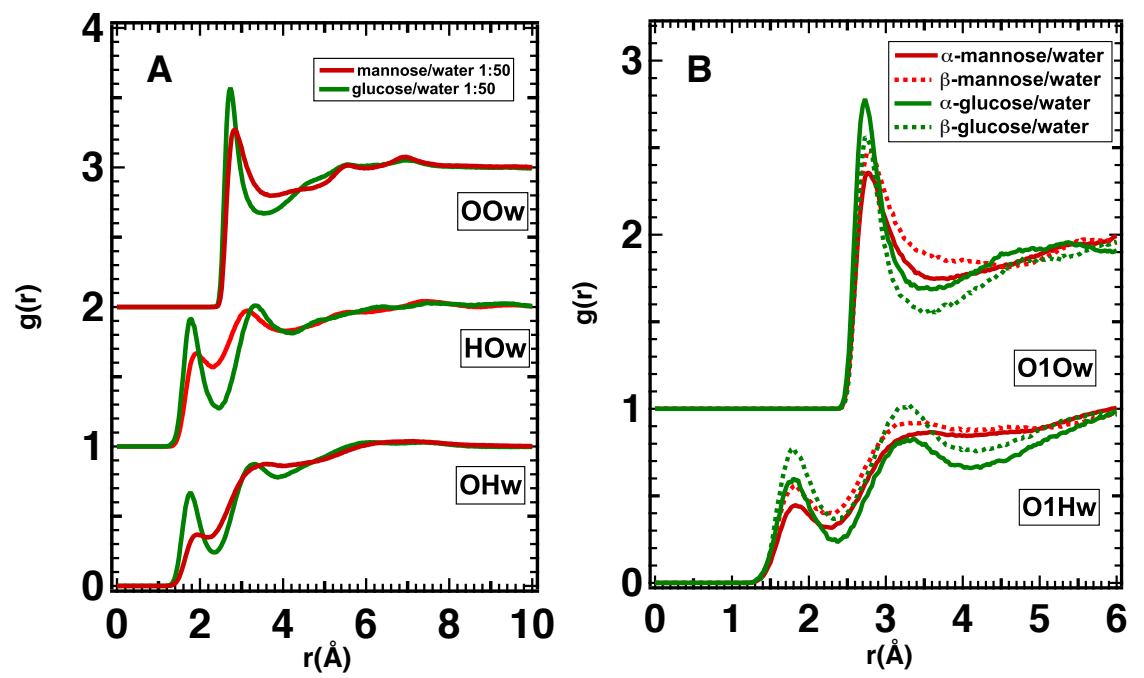

FIG. 4: The radial distribution functions of the O-Ow, O-Hw and H-Ow pairs. Data for mannose are reported in red, those for glucose in green; functions have been offset for clarity. A) Average hydration of $\mathrm{O} 3 \mathrm{H}, \mathrm{O} 4 \mathrm{H}, \mathrm{O} 5 \mathrm{H}$ and $\mathrm{O} 6 \mathrm{H}$ groups (configurations relative to both anomers have been averaged). B) Hydration of the $O 1$ sites: solid and dashed lines refer to the $\alpha$-and $\beta$-anomer, respectively. The hydration of the H1 sites (data not shown) shows similar differences between $\alpha$ and $\beta$-anomers.

data and did not show clear differences between the hydration of the two sugars.

The $g(r) \mathrm{s}$ for the hydration of the O1H1 hydroxyl, which distinguishes the $\alpha$ and $\beta$ anomers of the two monosaccharides, are shown in Fig. 4B. Similar to the other -OH groups, the first neighbor peaks are sharper and more intense for glucose, implying a higher directionality and number of HBs (the density of the two solutions is similar). Interestingly, for both sugars there are differences in the intensities of the first peaks in the $g(r)$ s for the $\alpha$ - and $\beta$-anomers, implying that the O1H1 group is more highly hydrated in the $\beta$-anomers. This observation suggests that the different position (below or above the ring plane, Fig. 1) of this -OH group cannot be solely responsible for the different taste of $\beta$ and $\alpha$ mannose. This observation is confirmed by the 3D arrangement of water molecules around the O1H1 groups and the other hydroxyl groups on the molecule, for instance the $\mathrm{O} 4 \mathrm{H}$ group shown 
in Fig. 5. Importantly, panels B and D confirm that in $\beta$-mannose and $\beta$-glucose the O1H1 sites are more highly hydrated than for their respective $\alpha$-anomer (panels A and $\mathrm{C}$ ), and that the regions where the probability of finding a water molecule is higher than 0.1 (red regions) are somewhat sharper for glucose (panels C,D), compared with those for mannose (panels A,B). In contrast, the same analysis for the $\mathrm{O} 4 \mathrm{H}$ hydroxyl on both sugars (plots $\mathrm{E}, \mathrm{F}, \mathrm{G}, \mathrm{H})$ shows a similar pattern for all four anomers.

The differences between the hydration shells of the two sugars, along with the different total number of HBs formed by glucose and mannose $(\sim 15$ compared to $\sim 10$, in agreement with 3) might explain the different taste between these molecules. However, the sweetness triangle and multipoint interaction models ${ }^{5,6,8}$ suggest that the spatial arrangement of donor/acceptor sites is relevant. To investigate this further, the contact coefficients of hydration shells for mannose and glucose for Ow around the - $\mathrm{OH}$ oxygens are shown in Fig. 6 (top panels). These show that the loss of hydration in mannose compared with glucose is a result of decreased hydration around the O3-O4-O5 hydroxyl oxygens, rather than around the O1H1 group which defines $\alpha$ amd $\beta$ anomers, and that this loss of hydration is comparatively more severe for $\beta$-mannose than for $\beta$-glucose. The contact coefficients for the $\mathrm{O} 6 \mathrm{H}$ group $\left(\mathrm{CH}_{2} \mathrm{OH}\right)$ show that this site is more hydrated in mannose than in glucose. However, this site is not directly involved in the AH-B-X binding model.

Finally, to better visualize the 3D arrangement of water molecules around the whole molecules, the SDFs are shown in Fig. 6 (bottom), which show some differences between the hydration shell of $\alpha$ - and $\beta$-anomers for both mannose and glucose. In particular, the hydration of $\beta$-mannose (Fig. 6B) is somewhat reduced around the O3, O4, O5 hydroxyl oxygens, in agreement with the reduced hydration around these sites observed in the contact coefficients. This suggests that, at odds with both glucose anomers and the $\alpha$ anomer of mannose, HBs from water molecules are not likely to occur to these hydroxyl oxygens, where consequently the interaction with the sweet receptor is in principle not favored. The $\alpha$-mannose hydration shell (Fig. 6A) covers instead an area with a spatial symmetry similar to that of $\alpha$-glucose (Fig. 6C), although the regions occupied by water molecules engaged in HBs are less well-defined and less populated compared to glucose. The hydration shells of the two glucose anomers have different shapes, but are equally well defined, implying that the number of HBs formed is similar and that both anomers have the same interaction capability with the sweet receptor. 
A

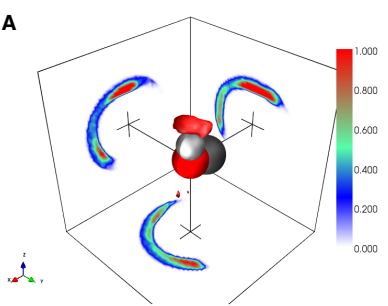

C
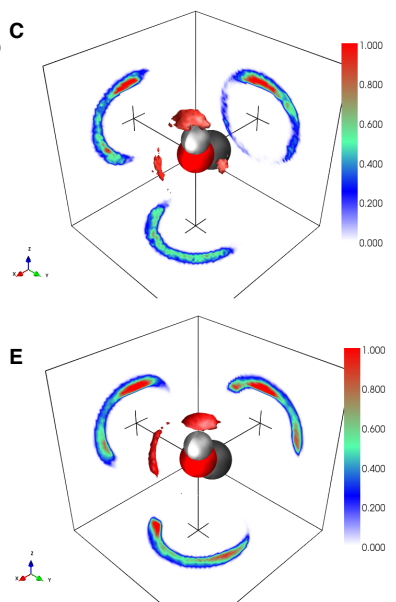

G

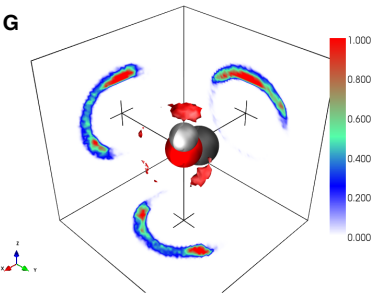

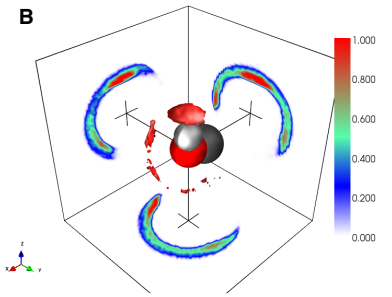
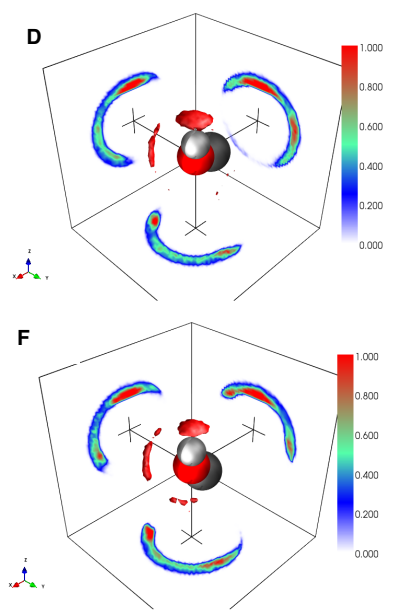

$\mathbf{H}$

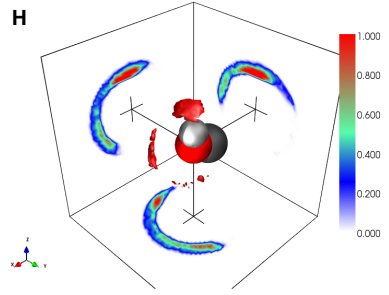

FIG. 5: SDMs of O1H1 (A,B,C,D) and O4H (E,F,G,H) groups of mannose (A,B,E,F) and glucose $(\mathrm{C}, \mathrm{D}, \mathrm{G}, \mathrm{H}): \alpha$ - anomers on the left and $\beta$ - ones on the right. The SDMs show the regions occupied by water molecules within a distance of $3.5 \AA$ from the $\mathrm{O} 1$ or $\mathrm{O} 4$ site, sitting at the origin of the reference frame, at the same isosurface fractional level of 0.1. For each SDM both cut throughs and heat maps are shown to emphasize the highest probability location for water molecules in each closest hydration shell. The cut throughs are taken through the origin, i. e. the oxygen atom, and are subsequently displaced by $6 \AA$ to the back for better visibility. 

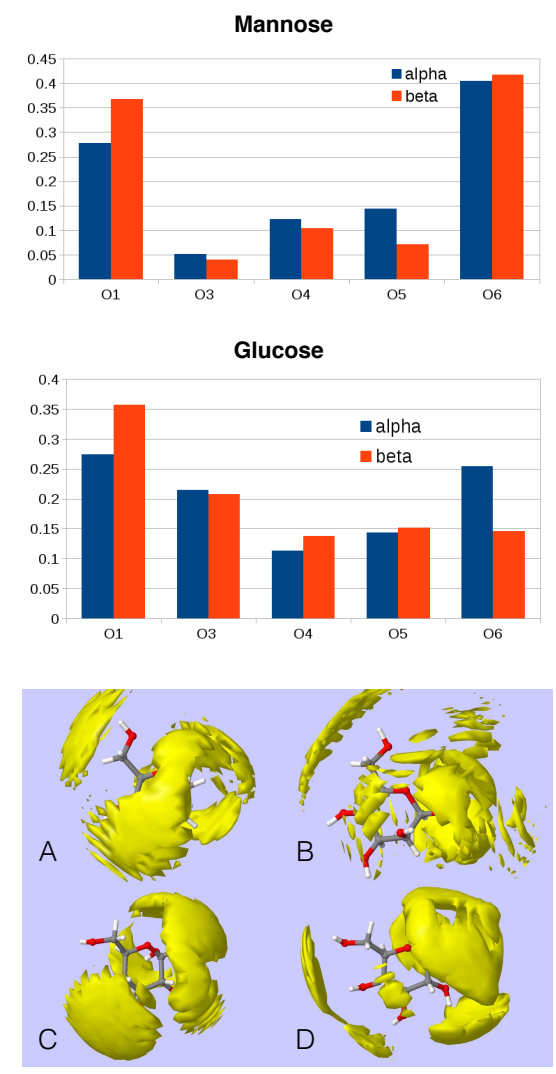

FIG. 6: (color online) The two top panels show the contact coefficients for water Ow atoms around the hydroxyl oxygens in all of the sugars investigated. For each sugar, the relative O-Ow contacts have been normalized to the total number of sugar-Ow contacts within a range of $0-3.5 \AA$. In the bottom panels the likely hydration of mannose and glucose is represented in 3D, in terms of SDFs. Panels A, B, C and D refer to $\alpha$ - and $\beta$ - anomers of mannose and glucose, respectively. The isosurfaces are shown at a fractional level of 0.04 , within distance of $10 \AA$ from the origin of the reference frame, sitting on the $\mathrm{O} 1$ atoms.

\section{Conclusions}

Although both sugars show regions where water is excluded, likely due to "hydrophobicity", glucose forms more and stronger hydrogen bonds with the surrounding water solvent 
compared to mannose. Indeed, while the glucose hydroxyls act as both HB donors and acceptors the mannose hydroxyls behave largely as donors. This, perhaps corresponds to the binding affinity and therefore sweetness of glucose compared with mannose. Although the binding affinity for mannose is unknown, a stronger binding affinity of a sugar is thought to correspond with it relative sweetness. ${ }^{32}$ and the results here suggest that in addition to mannose being less sweet, it likely binds with a lower affinity to the TIR2+T1R3 receptors.

More importantly, some major differences between the hydration shells of the four molecules are visible around the $\mathrm{O} 3 \mathrm{H}, \mathrm{O} 4 \mathrm{H}, \mathrm{O} 5 \mathrm{H}$ groups. While hydrating water molecules cover a thick "belt" around $\alpha$-glucose, this "belt" is thinner in $\alpha$-mannose, although still hydrating the $\mathrm{O} 3 \mathrm{H}, \mathrm{O} 4 \mathrm{H}, \mathrm{O} 5 \mathrm{H}$ groups. The symmetry of the hydration shell for $\beta$-anomers is different, nevertheless a hydration around the $\mathrm{O} 3 \mathrm{H}, \mathrm{O} 4 \mathrm{H}, \mathrm{O} 5 \mathrm{H}$ groups is still visible in $\beta$-glucose and almost absent in $\beta$-mannose. This suggests that both glucose anomers and $\alpha$-mannose expose a larger hydrogen bonding surface to the T1R2+T1R3 receptors than $\beta$ mannose. The hydration of the $\mathrm{O} 1 \mathrm{H} 1$ and $-\mathrm{CH}_{2} \mathrm{OH}$ groups in the four molecules are similar to each other and are not likely to aid in taste differences in vivo.

In conclusion, while all of these observations are consistent with both sweet taste receptor models and the relative sweetness of glucose and mannose, some questions remain; such as whether or not the sugar-receptor interaction is water mediated ${ }^{33}$. Additionally, the issue of the bitter aftertaste of aqueous solutions of mannose cannot be elucidated from the present results in the absence of a structural model for the T2R receptor.

\section{Acknowledgments}

The authors acknowledge useful discussion with Prof. M. Marino. This work has been performed within the Agreement No.0018318 (02/06/2014) between STFC and CNR, concerning collaboration in scientific research at the spallation neutron source ISIS and with partial financial support of CNR. This work has also be supported by funding from The Leverhulme Trust, UK (RPG-2015-135). Beamtime awarded by ISIS under RB number 
1520065 is gratefully acknowledged.

1 Chandrashekar,J.; Hoon, M. A.; Ryba, N. J. P.; Zuker, C. S.; The Receptors and Cells for Mammalian Taste Nature 2006, 444, 288-294.

2 Lerbret, A.; Bordat, P.; Affouard, F.; Guinet, Y.; Hedoux, A.; Paccou, L.; Prevost, D.; Descamps, M. Influence of Homologous Disaccharides on the Hydrogen-Bond Network of Water: Complementary Raman Scattering Experiments and Molecular Dynamics Simulations. Carbohydrate Res. 2005, 340, 881-887.

3 Shiraga, K.; Ogawa, Y.; Kondo, N.; Irisawa, A.; Imamura, M.. Evaluation of the Hydration State of Saccharides Using Terahertz Time-Domain Attenuated Total Reflection Spectroscopy. Food Chem. 2013, 140, 315-320.

4 Bouchard,A.; Hofland, G.W.; Witkamp, G.-J. Properties of Sugar, Polyol, and Polysaccharide Water-Ethanol Solutions. J. Chem. Eng. Data 2007, 52, 1838-1842.

5 Shallenberger, R. S.; Acree, T. E. Molecular Theory of Sweet Taste, Nature . 1967, 216, 480-482.

6 Kier, L. B. A Molecular Theory of Sweet Taste, J. Pharmaceutical Sci. 1972, 61, 1394-1397.

7 Eggers, S. C.; Acree, T. E.; Shallenberger, R. S. Sweetness Chemoreception Theory and Sweetness Transduction. Food Chem. 2000, 68, 45-49.

8 Nofre, C. ; Tinti, J.-M. Sweetness Reception in Man: the Multipoint Attachment Theory, Food Chemistry 1996, 56, 263-274.

9 Maugeri, L.; Busch, S.; McLain, S. E.; Pardo, L. C.; Bruni, F.; Ricci, M. A. StructureActivity Relationships in Carbohydrates Revealed by Their Hydration,BBA - General Subjects ihttp://doi.org/10.1016/j.bbagen.2016.12.017

10 Pagnotta, S. E.; McLain, S. E.; Soper, A. K.; Bruni, F.; Ricci, M. A. Water and trehalose: How much do they interact with each other? J. Phys. Chem. B 2010114 4904-4908.

11 Steinhardt, R. G. Jr; Calvin, A. D.; Dodd, E. A Taste-Structure Correlation with $\alpha$-D-Mannose and $\beta$-D-Mannose, Science 1962, 135, 367-8.

12 Stewart, R. A.; Carrico, C. K.; Webster, R. L.; Steinhardt, R. G. Physicochemical Stereospecificity in Taste Perception of $\alpha$-D-Mannose and $\beta$-D-Mannose, Nature 1971, 234, 220.

13 Tavagnacco, L.; Brady, J. W.; Bruni, F.; Callear, S.; Ricci, M. A.; Saboungi, M. L.; Cesaro, A. Hydration of Caffeine at High Temperature by Neutron Scattering and Simulation Studies, 
J.Phys. Chem. B, 2015,119, 13294-13301, DOI:10.1021/acs.jpcb.5b09204.

14 Busch, S.; Lorenz, C. D.; Taylor, J.; Pardo, L. C.; McLain, S. E.; Short-Range Interactions of Concentrated Proline in Aqueous Solution. J. Phys. Chem. B 2014, 11414267 - 14277.

15 Johnston, A.J., Busch, S.; Pardo, L.C.; Callear, S.K; Biggin, P.C.; McLain, S. E. On the Atomic Structure of Cocaine in Solution. Phys. Chem. Chem. Phys. 2016, 18991 - 999.

16 Steinke, N.; Genina, A.; Lorenz, C.D.; McLain, S.E. Salt Interactions in Solution Prevent Urea from Direct Association with a Peptide Backbone. J. Phys. Chem. B 2017, 1211866 - 1876.

17 O'Dell, W. B.; Baker, D.C.; McLain, S. E. Structural Evidence for Inter-Residue Hydrogen Bonding Observed for Cellobiose in Aqueous Solution. PLOS one. 2012, 7, e45311.

18 McLain, S. E.; Imberti, S.; Soper A. K.; Botti, A.; Bruni, F.; Ricci, M. A. Structure of 2 Molar $\mathrm{NaOH}$ in Aqueous Solution from Neutron Diffraction and Empirical Potential Structure Refinement, Phys. Rev. B, 2006, 74, 094201-8.

19 Soper, A. K. in Proceedings of the Conference on Advanced Neutron Sources 1988, IOP Conf. Proc. no. 97, Hyer, D. K. Editor (Institute of Physics and Physical Society, London, 1989) p.353.

20 http://www.isis.stfc.ac.uk/

21 Soper, A. K. in GudrunN and GudrunX: programs for correcting raw neutron and X-ray diffraction data to differential scattering cross section - July 2011, Rutherford Appleton Laboratory Technical Report, RAL-TR-2011-013.

22 V. F. Sears Neutron Scattering Lengths and Cross Sections, Neutron News 1992, 3, 26-37.

23 Soper, A. K. Computer Simulation as a Tool for the Interpretation of Total Scattering Data from Glasses and Liquids. Molecular Simulation, 2012, 38, 1171-1185.

24 Dabkowska, A. P.; Foglia, F.; Lawrence, M. J.; Lorenz, C. D.; McLain, S. E. On the Solvation Structure of Dimethylsulfoxide/Water Around the Phosphatidylcholine Head Group in Solution. J. Chem.Phys. 2011, 135, 225105.

25 Krautler, V; Muller, M; Hunenberger, P. H. Conformation, Dynamics, Solvation and Relative Stabilities of Selected $\beta$-Hexopyranoses in Water: a Molecular Dynamics Study with the GROMOS 45A4 Force Field, Carbohydrate Research 2007, 3422097 - 2124.

26 Towey, J . J.; Soper, A. K.; Dougan, L. Low-Density Water Structure Observed in a Nanosegregated Cryoprotectant Solution at Low Temperatures from 285 to $238 \mathrm{~K}$, J. Phys. Chem. B 2016, 1204439 - 4448. 
27 Berendsen, H. J. C.; Grigera,J. R.; Straatsma, T. P. The Missing Term in Effective Pair Potentials. J. Phys. Chem. 1987, 91, 6269-6271.

28 Postorino, P.; Nardone, M.; Ricci, M. A.; Rovere, M. Inelasticity Effects in the Neutron Diffraction Measurements from Water Steam Using Pulsed Sources, J. Molecular Liquids., 1995, 63, $221-240$

29 Pardo, L. C., M. Rovira-Esteva, J. L. Tamarit, N. Veglio, F. J. Bermejo, G. J. Cuello. In: Metastable Systems Under Pressure; Rzoska, S., Drozd-Rzoska, A., Mazur, V., Eds.; NATO Science for Peace and Security Series A:Chemistry and Biology; Springer: Dordrecht, Netherlands, 2010; pp $79-91$.

30 https://gcm.upc.edu/en/members/luis-carlos/angula/ANGULA

31 Henao, A. A.; Johnston, J.; Guàrdia, E.; McLain, S. E; Pardo, L.C. On the Positional and Orientational Order of Water and Methanol around Indole: a Study on the Microscopic Origin of Solubility. Phys. Chem. Chem. Phys. 2016, 18, 23006-23016.

32 Lum, C. K. L.; Henkin, R. I. Sugar Binding to Purified Fractions from Bovine Taste Buds and Epithelial Tissue. Bioch. Biophs. Acta 1976, 421, 380-394.

33 Maillet, E.L.; Cui, M.; Jiang, P.; Mezei, M.; Hecht, E.; Quijada, J.; Margolskee, R.F.; Osman, R.; Max, M. Characterization of the Binding Site of Aspartame in the Human Sweet Taste Receptor, Chem. Senses 201540 577-586. 


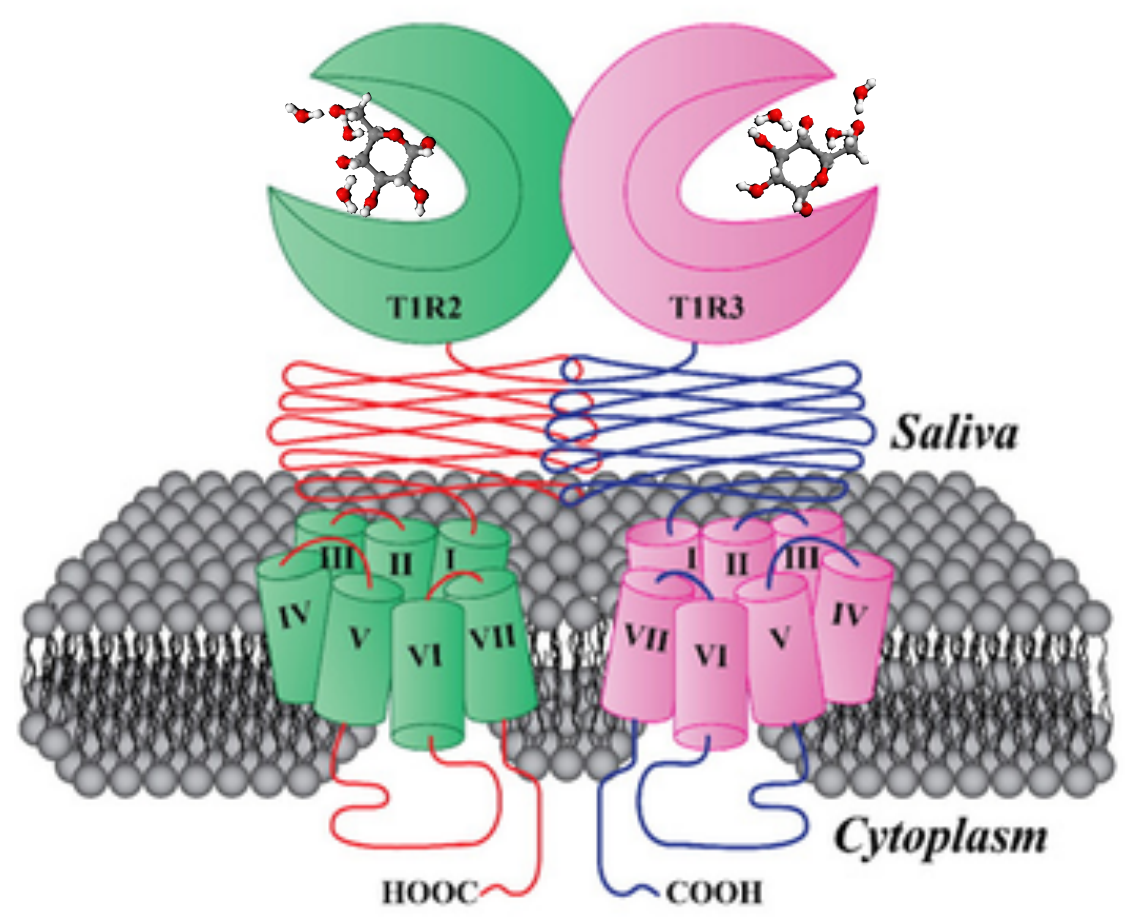

